International Mathematical Forum, 2, 2007, no. 44, 2161 - 2178

\title{
The Renormalization of Self Intersection Local Times of Fractional Brownian Motion
}

\author{
Anis Rezgui \\ Mathematics departement \\ INSAT centre urbain nord B.P. 676 \\ 1080 Tunis Tunisia \\ anis.rezgui@fsb.rnu.tn
}

\begin{abstract}
We present a new approach to treat the problem of self intersection local time of a d-dimensional Fractional Brownian motion based on the property of chaotic representation and the white noise analysis. This approach could be generalized to general Gaussian processes.
\end{abstract}

Mathematics Subject Classification: 60H40, 60J65

\section{Introduction}

Let $(\Omega, \mathcal{F}, \mathbb{P})$ be an abstract probability space which could be specified later and $\left\{B_{t}: t \geq 0\right\}$ a d-dimensional fractional Brownian Motion (fBm) with Hurst parameter $\left.H=\left(H_{1}, \cdots, H_{d}\right) \in\right] 0,1\left[^{d}\right.$.

It is known that

$$
B_{t}=\int_{0}^{t} K_{H}(t, s) d w_{s}
$$

where $\left\{w_{t}: t \geq 0\right\}$ is a standard Brownian motion and $K_{H}=\left(K_{H_{1}}, \cdots, K_{H_{d}}\right)$ is a kernel, (see for instance [16] for the proof).

We are interested, in this paper, in computing, when it exists, the selfintersection local time of the fBm. More precisely we study the existence of the limit when $\epsilon$ goes to zero, of the following sequence of processes

$$
L_{T}^{\epsilon}=\int_{0}^{T} \int_{0}^{t} P^{\epsilon}\left(B_{t}-B_{s}\right) d s d t s
$$

where

$$
P^{\epsilon}(x)=\frac{1}{\sqrt{2 \pi \epsilon}^{d}} e^{-\frac{|x|^{2}}{2 \epsilon}}, \quad x \in \mathbb{R}^{d} .
$$


Many questions arise here

1) Find out a relation between $N$ the number of chaos to be subtracted from $L_{T}^{\epsilon}$, d and $\mathrm{H}$ such that

$$
L_{T}^{\epsilon}-\sum_{n=0}^{N} L_{T, n}^{\epsilon}
$$

admits a limit in an appropriate space when $\epsilon \longrightarrow 0$.

2) Under which condition on $d$ and $H$ there is a blow up of the expectation $\mathbb{E}\left(L_{T}^{\epsilon}\right)$ when $\epsilon \longrightarrow 0$ ?

3) Suppose we are in the blow up case, find out a renormalization factor $r_{d, H}(\epsilon)$ such that

$$
r_{d, H}(\epsilon)\left(L_{T}^{\epsilon}-\mathbb{E}\left(L_{T}^{\epsilon}\right)\right)
$$

is bounded in $L^{2}(\Omega)$ when $\epsilon \longrightarrow 0$.

4) Find out the limit in $L^{2}(\Omega)$ or in law, when it exists, of

$$
r_{d, H}(\epsilon)\left(L_{T}^{\epsilon}-\mathbb{E}\left(L_{T}^{\epsilon}\right)\right)
$$

when $\epsilon \longrightarrow 0$.

In view of what has been done in the case of the classical Brownian motion, see for instance [3][8] [9] [11][17] and [18] we should compute explicitly the chaos of the $\mathrm{fBm}$, for this we need some tools from the white noise analysis.

\subsection{Tools from White Noise Analysis}

We quote some white noise analysis concepts as introduced in [3], referring to [10] for a systematic presentation. Consider a white noise space $\left(S^{\prime}(\mathbb{R})^{d}, \mathcal{B}, \mu\right)$, where $\mathcal{B}$ is the weak Borel $\sigma$-algebra of $S^{\prime}(\mathbb{R})^{d}$, and $\mu$ is the centered Gaussian measure whose covariance is given by the inner product of $L^{2}(\mathbb{R})^{d}$, in the sense that the vector valued white noise has the characteristic function

$$
C(\mathbf{f})=\mathbb{E}\left(e^{i\langle\omega, \mathbf{f}\rangle}\right)=\int_{S^{\prime}(\mathbb{R})^{d}} d \mu[\omega] e^{i\langle\omega, \mathbf{f}\rangle}=e^{-\frac{1}{2}\langle\mathbf{f}, \mathbf{f}\rangle},
$$

where $\langle\omega, \mathbf{f}\rangle=\sum_{j=1}^{d}\left\langle\omega_{j}, f_{j}\right\rangle$ and $f_{j} \in S(\mathbb{R}, \mathbb{R})$. Then a realization of a vector of independent fractional Brownian motions $B_{j}, j=1, \cdots, d$, is given by

$$
B_{j}(t)=\left\langle\omega_{j}, K_{H_{j}}\right\rangle=\int_{0}^{t} K_{H_{j}}(s, t) \omega_{j}(s) d s .
$$

We recall the explicit formula of the kernel of a one dimensional $\mathrm{fBm}$ with Hurst parameter $h \in] 0,1$ [, see for instance [1]

$h<1 / 2$

$$
K_{h}(t, u)=C_{h}\left\{\left(\frac{t}{2}\right)^{h-1 / 2}(t-u)^{h-1 / 2}-\right.
$$




$$
\left.(h-1 / 2) u^{1 / 2-h} \int_{u}^{t}(r-u)^{h-1 / 2} r^{h-3 / 2} d r\right\} 1_{[0, t]}(u),
$$

where $C_{h}$ is some constant.

$h>1 / 2$

$$
K_{h}(t, u)=C_{h} u^{1 / 2-h} \int_{u}^{t}(r-u)^{h-3 / 2} r^{h-1 / 2} d r 1_{[0, t]}(u)
$$

where $C_{h}$ is some constant.

Hence we consider independent d-tuples of Gaussian white noise $\omega=$ $\left(w_{1}, \cdots, \omega_{d}\right)$ and correspondingly, d-tuples of test functions $\mathbf{f}=\left(f_{1}, \cdots, f_{d}\right) \in$ $S\left(\mathbb{R}, \mathbb{R}^{d}\right)$, and use the following multi-index notation:

$$
\begin{gathered}
\vec{n} !=\prod_{1}^{d} n_{i} ! \\
\langle\mathbf{f}, \mathbf{f}\rangle=\sum_{i=1}^{d} \int d t f_{i}^{2}(t) \\
\left\langle F_{\vec{n}}, \mathbf{f}^{\otimes \vec{n}}\right\rangle=\int d^{n} t F_{\vec{n}}\left(t_{1}, \cdots, t_{n}\right) \bigotimes_{i=1}^{d} f_{i}^{\otimes n_{i}}\left(t_{1}, \cdots, t_{n}\right)
\end{gathered}
$$

and similarly for $\left\langle: \omega^{\otimes \vec{n}}:, F_{\vec{n}}\right\rangle$ where for d-tuples of white noise the Wick product : $\cdots$ : (see $[10])$ generalizes to

$$
: \omega^{\otimes \vec{n}}:=\bigotimes_{i=1}^{d}: \omega_{i}^{\otimes n_{i}}:
$$

The Hilbert space

$$
\left(L^{2}\right)=L^{2}(d \mu)
$$

is canonically isomorphic to the d-fold tensor product of Fock spaces of symmetric square integrable functions:

$$
\left(L^{2}\right) \simeq\left(\bigoplus_{k=0}^{\infty} \operatorname{SymL}^{2}\left(\mathbb{R}^{k}, k ! d^{k} t\right)\right)^{\otimes d}=\mathcal{F} .
$$

For a general element $\varphi$ of $\left(L^{2}\right)$ this implies the chaos expansion

$$
\varphi(\omega)=\sum_{\vec{n}=0}^{\infty}\left\langle: \omega^{\otimes \vec{n}}:, F_{\vec{n}}\right\rangle
$$

the norm of $\varphi$ is given by

$$
\|\varphi\|_{\left(L^{2}\right)}^{2}=\sum_{\vec{n}}^{\infty} \vec{n} !\left|F_{\vec{n}}\right|_{2, n}^{2}
$$


with kernel functions $F$ in $\mathcal{F}$ and where ||$_{2, n}$ is the norm in $L^{2}\left(\mathbb{R}^{n}, d t\right)$. Given $\xi \in S(\mathbb{R})^{d}$, let us consider the Wick exponential

$$
\begin{aligned}
: \exp \langle\omega, \xi\rangle: & \equiv \exp \left(\langle\omega, \xi\rangle-\frac{1}{2}(\xi, \xi)\right) \\
& =\sum_{\vec{n}} \frac{1}{\vec{n} !}\left\langle: \omega^{\otimes \vec{n}}:, \xi^{\otimes \vec{n}}\right\rangle, \quad \omega \in S^{\prime}(\mathbb{R})^{d} .
\end{aligned}
$$

The S-transform plays an important role in the study of stochastic processes in particular the computation of their chaos expansion, see for example [?][10]; we define the S-transform of $\varphi$ in $\left(L^{2}\right)$ as

$$
S \varphi(\xi) \equiv \ll \varphi,: \exp \langle., \xi\rangle: \gg=\sum_{\vec{n}}\left(\varphi_{\vec{n}}, \xi^{\otimes \vec{n}}\right)_{2, n}
$$

In particular, for Hermitian operators $\mathrm{A}$ in $L^{2}(\mathbb{R})$, we can define the "second quantization" of $A$ as an operator $\Gamma(A)$ in $\left(L^{2}\right)$ given by

$$
S \Gamma(A) \Phi(\cdot)=S \Phi(A \cdot)
$$

for $\Phi \in\left(L^{2}\right)$.

Generalized functions are obtained via a Gel'fand triple

$$
(S) \subset\left(L^{2}\right) \subset(S)^{*}
$$

with $(S)$ the projective limit Hilbert spaces $(S)_{k}$

$$
(S) \equiv \operatorname{projlim}_{k}(S)_{k} \quad \text { and } \quad(S)_{k} \equiv D\left(\Gamma\left(A^{k}\right)\right)
$$

where $A$ is operating on $L^{2}(\mathbb{R}, d u)$

$$
A f(u)=\left(-d^{2} / d u^{2}+u^{2}+1\right) f(u) .
$$

\section{The kernels of the self-intersection local time of the fBm}

$$
\begin{gathered}
L_{T}^{\epsilon}=\int_{0}^{T} \int_{0}^{t} P^{\epsilon}\left(B_{t}-B_{s}\right) d s d t \\
P^{\epsilon}(x)=\frac{1}{\sqrt{2 \pi \epsilon} d} e^{-\frac{|x|^{2}}{2 \epsilon}}=\frac{1}{(2 \pi)^{d}} \int_{\mathbb{R}^{d}} e^{i x \cdot \xi-\frac{\epsilon \xi^{2}}{2}} d \xi
\end{gathered}
$$

so

$$
L_{T}^{\epsilon}=\int_{0}^{T} \int_{0}^{t} d t d s \frac{1}{(2 \pi)^{d}} \int_{\mathbb{R}^{d}} e^{i\left(B_{t}-B_{s}\right) \cdot \xi-\frac{\epsilon \xi^{2}}{2}} d \xi
$$


in view of $(2)$, for $j=1, \cdots, d, B_{t}^{j}-B_{s}^{j}=\left\langle\Delta K_{j}, \omega_{j}\right\rangle$ with $\Delta K_{j}=K_{H_{j}}(u, t)-$ $K_{H_{j}}(u, s)$

$$
\begin{gathered}
S\left(e^{i\left(B_{t}-B_{s}\right) \cdot \xi}\right)(f)=\prod_{j=1}^{d} S\left(e^{i\left\langle\Delta K_{j}, \omega_{j}\right\rangle \xi_{j}}\right)\left(f_{j}\right) \\
S\left(e^{i\left\langle\Delta K_{j}, \omega_{j}\right\rangle \xi_{j}}\right)\left(f_{j}\right)=e^{-\frac{\left|f_{j}\right|_{2}^{2}}{2}} \int_{S^{\prime}(\mathbb{R})} d \gamma_{j}\left(\omega_{j}\right) e^{i\left\langle\Delta K_{j}, \omega_{j}\right\rangle \xi_{j}+\left\langle f_{j}, \omega_{j}\right\rangle} \\
=e^{-\frac{\left|f_{j}\right|_{2}^{2}}{2}} \int_{S^{\prime}(\mathbb{R})} d \gamma_{j}\left(\omega_{j}\right) e^{i\left\langle\xi_{j} \Delta K_{j}-i f_{j}\right\rangle} \\
=e^{-\frac{\xi_{j}^{2}\left|\Delta K_{j}\right|_{2}^{2}}{2}} e^{i \xi_{j}\left\langle\Delta k_{j}, f_{j}\right\rangle}
\end{gathered}
$$

The last equality was by definition of $\gamma$ and so

$$
\frac{1}{(2 \pi)^{d}} \int_{\mathbb{R}^{d}} d \xi e^{-\frac{\epsilon \xi^{2}}{2}} S\left(e^{i\left(B_{t}-B_{s}\right) \cdot \xi}\right)(f)=\frac{1}{(2 \pi)^{d}} \int_{\mathbb{R}^{d}} \prod_{j=1}^{d} e^{-\frac{\xi_{j}^{2}}{2}\left(\left|\Delta K_{j}\right|_{2}^{2}+\epsilon\right)} e^{i \xi_{j}\left\langle\Delta k_{j}, f_{j}\right\rangle} d \xi_{j}
$$

using this elementary equality

$$
\int_{\mathbb{R}} e^{-\frac{\alpha x^{2}}{2}+i \beta x}=\sqrt{\frac{2 \pi}{\alpha}} e^{-\frac{\beta^{2}}{2 \alpha}}
$$

we obtain

$$
\begin{aligned}
S P^{\epsilon}\left(B_{t}-B_{s}\right)(f) & =\frac{1}{\sqrt{2 \pi}^{d}} \prod_{j=1}^{d} \frac{1}{\sqrt{\left|\Delta K_{j}\right|_{2}^{2}+\epsilon}} \exp \left\{-\frac{\left\langle f_{j}, \Delta K_{j}\right\rangle^{2}}{2\left(\left|\Delta K_{j}\right|_{2}^{2}+\epsilon\right)}\right\} \\
& =\frac{1}{\sqrt{2 \pi}^{d}} \prod_{j=1}^{d} \frac{1}{\sqrt{|t-s|^{2 h_{j}+\epsilon}}} \exp \left\{-\frac{\left\langle f_{j}, \Delta K_{j}\right\rangle^{2}}{2\left(|t-s|^{2 H_{j}}+\epsilon\right)}\right\} \\
& =\frac{1}{\sqrt{2 \pi}^{d}} \prod_{j=0}^{d} \sum_{n_{j}=0}^{\infty} \frac{1}{n_{j} !}\left(-\frac{1}{2}\right)^{n_{j}} \frac{1}{\left(|t-s|^{2 H_{j}}+\epsilon\right)^{n_{j}+1 / 2}}\left\langle f_{j}, \Delta K_{j}\right\rangle^{2 n_{j}} \\
& =\frac{1}{\sqrt{2 \pi}^{d}} \sum_{\vec{n}=0}^{\infty} \frac{1}{\vec{n} !}\left(-\frac{1}{2}\right)^{n} \prod_{j=1}^{d}\left(\frac{1}{\left(|t-s|^{2 H_{j}}+\epsilon\right)^{n_{j}+1 / 2}}\right)\left\langle f^{\otimes 2 \vec{n}}, \Delta K^{\otimes 2 \vec{n}}\right\rangle .
\end{aligned}
$$

Finally we obtain

Proposition 1 Let $\vec{n} \in \mathbb{N}^{d}$ and $d \geq 1$,

$$
L_{T}^{\epsilon}=\sum_{\vec{n}=0}^{\infty} L_{T, 2 \vec{n}}^{\epsilon}=\frac{1}{\sqrt{2 \pi} d} \sum_{\vec{n}=0}^{\infty} \frac{1}{\vec{n} !}\left(-\frac{1}{2}\right)^{n}\left\langle: \omega^{\otimes 2 \vec{n}}:, l_{T, 2 \vec{n}}^{\epsilon}\right\rangle
$$

then

$$
l_{T, 2 \vec{n}}^{\epsilon}=\int_{0}^{T} d t \int_{0}^{t} d s \prod_{j=1}^{d}\left(\frac{1}{\left(|t-s|^{2 H_{j}}+\epsilon\right)^{n_{j}+1 / 2}}\right) \Delta K^{\otimes 2 \vec{n}}
$$




\subsection{The expectation of the self-intersection local time of the $\mathrm{fBm}$}

Let us now compute the expectation of the self-intersection local time of the $\mathrm{fBm}, \mathbb{E}\left(L_{T}^{\epsilon}\right)$, it is just the first chaos, so in view of the last proposition

$$
\begin{aligned}
\mathbb{E}\left(L_{T}^{\epsilon}\right) & =\frac{1}{\sqrt{2 \pi}^{d}} \int_{0}^{T} d t \int_{0}^{t} d s \prod_{j=1}^{d}\left(\frac{1}{\left(|t-s|^{2 H_{j}}+\epsilon\right)^{1 / 2}}\right) \\
& =\frac{1}{\sqrt{2 \pi}^{d}} \int_{0}^{T} d s \frac{T-s}{\prod_{j=1}^{d}\left(s^{2 H_{j}}+\epsilon\right)^{1 / 2}}
\end{aligned}
$$

we use the change of variables $s=\epsilon^{\frac{d}{2 H^{*}}} z=\alpha(\epsilon) z$ with $H^{*}=\sum_{j=1}^{d} H_{j}$

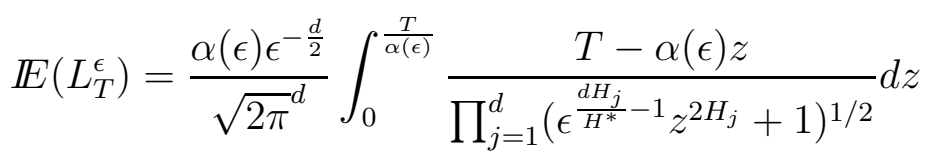

we divide the integral in two parts

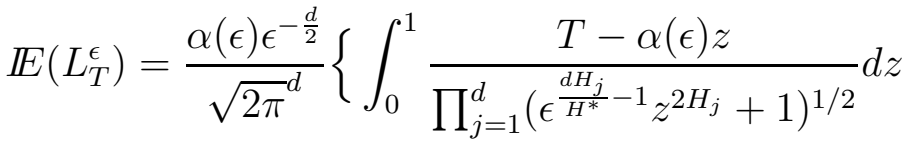

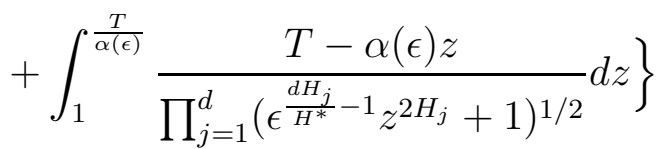

the first integral in braces is bounded.

Set $\pi$ the second integral in braces, so

$$
\pi \leq T \int_{1}^{\frac{T}{\alpha(\epsilon)}} \frac{d z}{z^{H^{*}}}= \begin{cases}T^{1-H^{*}} \epsilon^{\frac{d}{2}-\frac{d}{2 H^{*}}}-1 & H^{*} \neq 1 \\ \log T-\frac{d}{2 H^{*}} \log \epsilon & H^{*}=1\end{cases}
$$

Proposition 2 Let $T>0$ and $d \geq 1$.

If $H^{*} \geq 1$

$$
\lim _{\epsilon \rightarrow 0} \mathbb{E}\left(L_{T}^{\epsilon}\right)=\infty
$$

Moreover if $H^{*}=1$

$$
\mathbb{E}\left(L_{T}^{\epsilon}\right) \leq \text { const. } \times|\log \epsilon|,
$$

and if $H^{*}>1$

$$
\mathbb{E}\left(L_{T}^{\epsilon}\right) \leq \text { const. } \times \epsilon^{\frac{d}{2}-\frac{d}{2 H^{*}}}
$$

where the constants depend on $T, H^{*}$ and $d$.

If $H^{*}<1$ there is no blow up

$$
\lim _{\epsilon \longrightarrow 0} \mathbb{E}\left(L_{T}^{\epsilon}\right)=\frac{1}{\sqrt{2 \pi}^{d}} \int_{0}^{T} d s \frac{T-s}{s^{H^{*}}} .
$$


Proposition 3 Suppose all $H_{j}=H$. Let $T>0$ and $d \geq 1$.

If $H^{*}=d H=1$

$$
\mathbb{E}\left(L_{T}^{\epsilon}\right)=\frac{1}{2 H}|\log \epsilon|(1+o(1))
$$

If $d H>1$

$$
\mathbb{E}\left(L_{T}^{\epsilon}\right)=C_{H} \epsilon^{\frac{1}{2 H}-\frac{d}{2}}(1+o(1))
$$

where $C_{H}=\int_{0}^{\infty} \frac{d s}{\left(s^{2 H}+1\right)^{d / 2}}$.

\section{Remark}

If all $H_{j}=\frac{1}{2}$ and $d=2$ we are in the case $H^{*}=1$ and we obtain the Varadhan renormalization term [23].

\section{The self-intersection local time of the $\mathrm{fBm}$ as a generalized function}

First of all let us recall

Theorem 1. [9]

Let $(\Omega, \mathcal{F}, m)$ be a measure space, and $\Phi_{\lambda}$ a mapping defined on $\Omega$ with values in $(S)^{*}$. We assume that the $S$-transform of $\Phi_{\lambda}$

(1) is an m-measurable function of $\lambda$ for any test function $f \in S(\mathbb{R})^{d}$

(2) obeys to the following estimate

$$
\left|S \Phi_{\lambda}(f)\right| \leq C_{1}(\lambda) \exp \left\{C_{2}(\lambda)\left|A^{p} f\right|_{2}^{2}\right\}
$$

for some fixed $p$ and for $C_{1} \in L^{1}(m), C_{2} \in L^{\infty}(m)$.

Then $\Phi_{\lambda}$ is Bochner-integrable in the Hilbert space $(S)_{-q}$ for q large enough,

$$
\int_{\Omega} d m(\lambda) \Phi_{\lambda} \in(S)^{*}
$$

and

$$
S\left(\int_{\Omega} d m(\lambda) \Phi_{\lambda}\right)(f)=\int_{\Omega} d m(\lambda) S \Phi_{\lambda}(f) .
$$

Using the last theorem and the following "formula"

$$
\delta "=" \frac{1}{(2 \pi)^{d}} \int_{\mathbb{R}^{d}} e^{i \xi \cdot x} d \xi
$$


we could define $\delta\left(B_{t}-B_{s}\right)$ as a Bochner integral in $(S)^{*}$ and

$$
S\left(\delta\left(B_{t}-B_{s}\right)\right)(f)=\frac{1}{\sqrt{2 \pi}^{d}} \frac{1}{|t-s|^{H^{*}}} \prod_{j=1}^{d} \exp \left\{-\frac{\left\langle f_{j}, \Delta K_{j}\right\rangle^{2}}{|t-s|^{2 H_{j}}}\right\}
$$

And so, again by the last theorem, if $H^{*}<1$

$$
L_{T}=\int_{0}^{T} \int_{0}^{t} d s d t \delta\left(B_{t}-B_{s}\right)
$$

is well defined in $(S)^{*}$.

Suppose now that $H^{*} \geq 1$.

The idea is that if we subtract some of the first terms in the expansion of the exponential function in the expression of the S-transform of $\delta\left(B_{t}-B_{s}\right)$, we could obtain an integrable function in factor of the remaining part, then the second condition of Theorem 1 will be satisfied.

And so we could define a renormalization of the self-intersection local time in $(S)^{*}$.

Let $N \in \mathbb{N}$ and define $\delta^{(2 N)}\left(B_{t}-B_{s}\right)$ by its S-transform

$$
S \delta^{(2 N)}\left(B_{t}-B_{s}\right)(f)=\frac{1}{\sqrt{2 \pi}^{d}} \frac{1}{|t-s|^{H^{*}}} \sum_{\vec{n}, n \geq N}\left(\frac{-1}{2}\right)^{n} \prod_{j=1}^{d} \frac{\left\langle f_{j}, \Delta K_{j}\right\rangle_{2}^{2 n_{j}}}{|t-s|^{2 H_{j} n_{j}}}
$$

SO

$$
\left|S \delta^{(2 N)}\left(B_{t}-B_{s}\right)(f)\right| \leq \frac{1}{\sqrt{2 \pi}^{d}} \frac{1}{|t-s|^{H^{*}}} \sum_{\vec{n}, n \geq N} \frac{1}{2^{n}} \prod_{j=1}^{d} \frac{\left|f_{j}\right|_{\infty}^{2 n_{j}}\left|\Delta K_{j}\right|_{1}^{2 n_{j}}}{|t-s|^{2 H_{j} n_{j}}}
$$

we need to estimate the $L^{1}$-norm of $\Delta K_{j},\left|\Delta K_{j}\right|_{1}$ for fixed $\mathrm{j}$.

1) We treat first the case when all $H_{j}>1 / 2$.

Let $h>1 / 2$, in view of $(4)$

$$
\begin{gathered}
\Delta K_{h}=K_{h}(t, u)-K_{h}(s, u)=C_{h} u^{1 / 2-h}\left\{1_{[0, s]}(u) \int_{s}^{t}(r-u)^{h-3 / 2} r^{h-1 / 2} d r\right. \\
\left.+1_{[s, t]}(u) \int_{u}^{t}(r-u)^{h-3 / 2} r^{h-1 / 2} d r\right\}
\end{gathered}
$$

we obtain

$$
\left|\Delta K_{h}\right|_{1} \leq C_{h}\left\{c_{1} T^{h-1 / 2}|t-s|+c_{2}|t-s|^{h+1 / 2}\right\}
$$

suppose that $|t-s|$ is small enough, we get

$$
\begin{aligned}
\left|S \delta^{(2 N)}\left(B_{t}-B_{s}\right)(f)\right| & \leq \frac{1}{\sqrt{2 \pi}^{d}} \frac{1}{|t-s|^{H^{*}}} \sum_{\vec{n}, n \geq N} \frac{1}{2^{n}}|t-s|^{2 n} \prod_{j=1}^{d} \frac{1}{|t-s|^{2 H_{j} n_{j}}} \prod_{j=1}^{d}\left|f_{j}\right|_{\infty}^{2 n_{j}} \\
& \leq \frac{1}{\sqrt{2 \pi}^{d}} \frac{1}{|t-s|^{H^{*}+2 N(\bar{H}-1)}} \exp \left\{\frac{1}{2} \sum_{j=1}^{d}\left|f_{j}\right|_{\infty}^{2}\right\}
\end{aligned}
$$


where $\bar{H}=\max _{j} H_{j}$.

Theorem 2. Let $T>0$ and $N \in \mathbb{N}$, suppose that

$$
2 N>\frac{H^{*}-1}{1-\bar{H}}
$$

then

$$
L_{T}^{(2 N)} \equiv \int_{0}^{T} \int_{0}^{t} d s d t \delta^{(2 N)}\left(B_{t}-B_{s}\right)
$$

is well defined as an element of $(S)^{*}$ and

$$
\lim _{\epsilon \longrightarrow 0} L_{\epsilon}^{(2 N)}=L^{(2 N)} \quad \text { in }(S)^{*}
$$

2) When all $H_{j}<1 / 2$ we obtain a bad estimation of $\left|\Delta K_{j}\right|_{1}$ and so we don't have a result.

\section{Remark}

When all $H_{j}=1 / 2$, the condition under which $L^{(2 N)}$ is well defined in $(S)^{*}$ is that $2 N>d-2$, this correspond to a result obtained in [9].

\section{Estimation of the $L^{2}$-norms of the chaos of the $\mathrm{fBm}$}

Suppose, in this section that we are in the blow up case i.e $H^{*} \geq 1$ and that $H<\frac{1}{2}$. Now we state our main result

Theorem 3. Let $T>0, \vec{n} \neq 0$ and $d \geq 2$. Then, if $d H=1$

$\frac{1}{\sqrt{|\log \epsilon|}}\left|L_{T, 2 \vec{n}}^{\epsilon}\right|_{2}$ has a finite non trivial limit when $\epsilon$ goes to zero and

$$
\lim _{\epsilon \rightarrow 0} \frac{1}{\sqrt{|\log \epsilon|}}\left|L_{T, 2 \vec{n}}^{\epsilon}\right|_{2} \leq \frac{1}{\sqrt{2 \pi}^{d}} \frac{\sqrt{2} T \sqrt{(2 \vec{n}) !}}{(\vec{n} !)} b(H, d, n)^{1 / 2} .
$$

If $d H \in] 1,3 / 2[$,

$$
\lim _{\epsilon \rightarrow 0}\left|L_{2 \vec{n}}^{\epsilon}\right| \frac{1}{\sqrt{2 \pi}^{d}} \frac{\sqrt{(2 \vec{n}) !}}{(\vec{n} !)} \frac{c(H, d, n)^{1 / 2}}{\sqrt{(3-2 H d)(2-H d)}} T^{2-H d} .
$$


If $d H=3 / 2$

$\frac{1}{\sqrt{|\log \epsilon|}}\left|L_{T, 2 \vec{n}}^{\epsilon}\right|_{2}$ has a finite non trivial limit when $\epsilon$ goes to zero and

$$
\lim _{\epsilon \rightarrow 0} \frac{1}{\sqrt{|\log \epsilon|}}\left|L_{T, 2 \vec{n}}^{\epsilon}\right|_{2} \leq \frac{1}{\sqrt{2 \pi}^{d}} \sqrt{\frac{T}{H}} \frac{\sqrt{(2 \vec{n}) !}}{(\vec{n} !)} c(H, d, n)^{1 / 2} .
$$

If $d H>3 / 2$

$$
\lim _{\epsilon \rightarrow 0} \epsilon^{\frac{d}{2}-\frac{3}{4 H}}\left|L_{2 \vec{n}}^{\epsilon}\right|_{2}=\frac{1}{\sqrt{2 \pi}^{d}} \frac{\sqrt{2 T} \sqrt{2 \vec{n} !}}{\vec{n} ! 2^{n}}(f(H, d, n)+g(H, d, n))^{1 / 2},
$$

where the constants $b, c, f$ and $g$ are given in (21), (23), (19) and (22)

\section{Remark}

1)The results of the last theorem generalizes the case of the classical Brownian motion i.e $H=1 / 2$, in fact it was shown in [3] that the renormalization factor was $|\log \epsilon|^{-1 / 2}$ when $d=3$ and $\epsilon^{\frac{d-3}{2}}$ when $d \geq 4$.

2)It was shown in [21] that when $d=2$ and $1 / d<H<\frac{3}{2 d}$ there is no need to renormalize by multiplication, the result in our theorem could be seen as a generalization for $H<1 / 2$ and $d \geq 2$.

3) The case $H>1 / 2$ is more complicated because we obtain a specific singularity in $\epsilon$ for each chaos. Which is not so surprising because when $H>1 / 2$ the $\mathrm{fBm}$ is smoother and, intuitively, its self intersection local time is worse.

\section{Proof of Theorem 3}

For $\vec{n} \in \mathbb{N}^{d}$ the $2 \vec{n}$ th chaos is given by (see proposition 1 )

$$
\begin{gathered}
L_{T, 2 \vec{n}}^{\epsilon}=\frac{1}{\sqrt{2 \pi}^{d}} \frac{1}{\vec{n} !}\left(-\frac{1}{2}\right)^{n}\left\langle: \omega^{\otimes 2 \vec{n}}:, l_{T, 2 \vec{n}}^{\epsilon}\right\rangle \\
l_{T, 2 \vec{n}}^{\epsilon}=\int_{0}^{T} d t \int_{0}^{t} d s \prod_{j=1}^{d}\left(\frac{1}{\left(|t-s|^{2 H_{j}}+\epsilon\right)^{n_{j}+1 / 2}}\right) \Delta K^{\otimes 2 \vec{n}}
\end{gathered}
$$

SO

$$
\mathbb{E}\left\{\left(L_{T, 2 \vec{n}}^{\epsilon}\right)^{2}\right\}=\frac{1}{(2 \pi)^{d}} \frac{1}{(\vec{n} !)^{2}}\left(\frac{1}{2}\right)^{2 n}(2 \vec{n}) !\left|l_{T, 2 \vec{n}}^{\epsilon}\right|_{L^{2}\left(\mathbb{R}^{2 n}\right)}^{2}
$$

In view of (17), we need to estimate $\left|l_{T, 2 \vec{n}}^{\epsilon}\right|_{\mathbb{R}^{2 n}}^{2}=\left|l_{T, 2 \vec{n}}^{\epsilon}\right|_{2,2 n}^{2}$,

$$
\begin{gathered}
\left|l_{T, 2 \vec{n}}^{\epsilon}\right|_{2,2 n}^{2}= \\
\int_{\mathbb{R}^{2 n}} d^{2 n} u \int_{0}^{T} \int_{0}^{t} \int_{0}^{T} \int_{0}^{t^{\prime}} d t d s d t^{\prime} d s^{\prime} \prod_{j=1}^{d} \frac{\Delta K_{j}(t, s)^{\otimes 2 n_{j}}(t, s) \Delta K_{j}(t, s)^{\otimes 2 n_{j}}\left(t^{\prime}, s^{\prime}\right)}{\left(\left(|t-s|^{2 H_{j}}+\epsilon\right)\left(\left|t^{\prime}-s^{\prime}\right|^{2 H_{j}}+\epsilon\right)\right)^{n_{j}+1 / 2}}
\end{gathered}
$$


by using Fubini theorem we first get

$$
\begin{aligned}
& \int_{\mathbb{R}^{2 n}} d^{2 n} u \prod_{j=1}^{d} \Delta K_{j}(t, s)^{\otimes 2 n_{j}}(t, s) \Delta K_{j}(t, s)^{\otimes 2 n_{j}}\left(t^{\prime}, s^{\prime}\right)= \\
& =\prod_{j=1}^{d} \int_{\mathbb{R}^{2 n_{j}}} d^{2 n_{j}} u \Delta K_{j}(t, s)^{\otimes 2 n_{j}}(t, s) \Delta K_{j}(t, s)^{\otimes 2 n_{j}}\left(t^{\prime}, s^{\prime}\right) \\
& =\prod_{j=1}^{d} \prod_{i=1}^{2 n_{j}} \int_{\mathbb{R}} d u_{i}^{j} \Delta K_{j}(t, s)(t, s) \Delta K_{j}(t, s)\left(t^{\prime}, s^{\prime}\right) \\
& =\prod_{j=1}^{d} \prod_{i=1}^{2 n_{j}} \mathbb{E}\left(\Delta B_{j}(t, s) \Delta B_{j}\left(t^{\prime}, s^{\prime}\right)\right) \\
& =\prod_{j=1}^{d}\left(\left|t-t^{\prime}\right|^{2 H_{j}}+\left|s-s^{\prime}\right|^{2 H_{j}}-\left|t-s^{\prime}\right|^{2 H_{j}}-\left|s-t^{\prime}\right|^{2 H_{j}}\right)^{2 n_{j}}
\end{aligned}
$$

so

$$
\begin{gathered}
\left|l_{T, 2 \vec{n}}^{\epsilon}\right|_{2,2 n}^{2}=\int_{0}^{T} \int_{0}^{t} \int_{0}^{T} \int_{0}^{t^{\prime}} d t d s d t^{\prime} d s^{\prime} \times \\
\prod_{j=1}^{d} \frac{\left(\left|t-t^{\prime}\right|^{2 H_{j}}+\left|s-s^{\prime}\right|^{2 H_{j}}-\left|t-s^{\prime}\right|^{2 H_{j}}-\left|s-t^{\prime}\right|^{2 H_{j}}\right)^{2 n_{j}}}{\left(\left(|t-s|^{2 H_{j}}+\epsilon\right)\left(\left|t^{\prime}-s^{\prime}\right|^{2 H_{j}}+\epsilon\right)\right)^{n_{j}+1 / 2}}
\end{gathered}
$$

in view of the symmetry of the domain and the integrand function it suffices to integrate only on $T_{1} \cup T_{2}$ where $T_{1}=\left\{0<s^{\prime}<t^{\prime}<s<t<T\right\}$ and $T_{2}=\left\{0<s^{\prime}<s<t^{\prime}<t<T\right\}$.

Let us first integrate over $T_{1}$, we make the following change of variables

$$
\left\{\begin{array}{l}
x=t-s \\
y=t^{\prime}-s^{\prime} \\
z=s-t^{\prime}
\end{array}\right.
$$

where $\mathrm{t}$ is considered as a parameter. Set $\left|l_{T, 2 \vec{n}}^{\epsilon, 1}\right|_{2,2 n}^{2}\left(\operatorname{resp} .\left|l_{T, 2 \vec{n}}^{\epsilon, 2}\right|_{2,2 n}^{2}\right)$ the integral over $T_{1}$ (resp. $T_{2}$ ), we obtain

$$
\begin{gathered}
\left|l_{T, 2 \vec{n}}^{\epsilon, 1}\right|_{2,2 n}^{2}=\int_{0}^{T} d t \int_{0 \leq x+y+z \leq t} d x d y d z \times \\
\prod_{j=1}^{d} \frac{\left.\left((x+z)^{2 H_{j}}+(y+z)^{2 H_{j}}-(x+y+z)^{2 H_{j}}-z^{2 H_{j}}\right)^{2 n_{j}}\right)}{\left(\left(x^{2 H_{j}}+\epsilon\right)\left(y^{2 H_{j}}+\epsilon\right)\right)^{n_{j}+1 / 2}}
\end{gathered}
$$


it is almost impossible to compute this integral (at least for us) when all $H_{j}$ are different, so let us suppose that all $H_{j}$ are equal to some $H$.

Denote by $\theta_{t}(\epsilon)=\frac{t}{\epsilon \frac{1}{2 H}}$, and make the following change of variables, $(x, y, z)=$ $\epsilon^{\frac{1}{2 H}}\left(x^{\prime}, y^{\prime}, z^{\prime}\right)$, we get

$$
\begin{aligned}
& \left|l_{T, 2 \vec{n}}^{\epsilon, 1}\right|_{2,2 n}^{2}=\epsilon^{\frac{3}{2 H}-d} \int_{0}^{T} \int_{0 \leq x+y+z \leq \theta_{t}(\epsilon)} d x d y d z \times \\
& \frac{\left((x+z)^{2 H}+(y+z)^{2 H}-(x+y+z)^{2 H}-z^{2 H}\right)^{2 n}}{\left(\left(x^{2 H}+1\right)\left(y^{2 H}+1\right)\right)^{n+d / 2}},
\end{aligned}
$$

by a symmetry argument in $(x, y)$

$$
\begin{aligned}
& \left|l_{T, 2 \vec{n}}^{\epsilon, 1}\right|_{2,2 n}^{2}=2 \epsilon^{\frac{3}{2 H}-d} \int_{0}^{T} \int_{\substack{0 \leq x+y+z \leq \theta_{t}(\epsilon) \\
0 \leq x \leq y \leq \theta_{t}(\epsilon)}} d x d y d z \times \\
& \frac{\left((x+z)^{2 H}+(y+z)^{2 H}-(x+y+z)^{2 H}-z^{2 H}\right)^{2 n}}{\left(\left(x^{2 H}+1\right)\left(y^{2 H}+1\right)\right)^{n+d / 2}}
\end{aligned}
$$

denote by

$$
f_{H}(x, y, z)=(x+z)^{2 H}+(y+z)^{2 H}-(x+y+z)^{2 H}-z^{2 H}
$$

and by

$$
f(t, H, d, n, \epsilon)=\int_{\substack{0 \leq x+y+z \leq \theta_{t}(\epsilon) \\ 0 \leq x \leq y \leq \theta_{t}(\epsilon)}} d x d y d z \frac{\left(f_{H}(x, y, z)\right)^{2 n}}{\left(\left(x^{2 H}+1\right)\left(y^{2 H}+1\right)\right)^{n+d / 2}} .
$$

First, note that $\left|f_{H}(x, y, z)\right| \leq x^{2 H}$ and for $d H>1$

$$
\int_{0 \leq x \leq y} d x d y \frac{x^{4 H n}}{\left(\left(x^{2 H}+1\right)\left(y^{2 H}+1\right)\right)^{n+d / 2}}<\infty
$$

this implies that for every $z \in \mathbb{R}_{+}$

$$
\int_{0 \leq x \leq y} d x d y \frac{f_{H}(x, y, z)^{2 n}}{\left(\left(x^{2 H}+1\right)\left(y^{2 H}+1\right)\right)^{n+d / 2}}
$$


exists. On the other hand for $\alpha \in] 1,2-2 H\left[, z^{\alpha} f_{H}(x, y, z)^{2 n}\right.$ decreases to zero when $\mathrm{z}$ tends to infinity, then

$$
f(H, d, n)=\int_{\mathbb{R}_{+}} d z \int_{0 \leq x \leq y} d x d y \frac{f_{H}(x, y, z)^{2 n}}{\left(\left(x^{2 H}+1\right)\left(y^{2 H}+1\right)\right)^{n+d / 2}}<\infty
$$

and so that

$$
\lim _{\epsilon \rightarrow 0} f(t, H, d, n, \epsilon)=f(H, d, n) .
$$

Therefore we obtain, if $d H \in] 1,3 / 2[$

$$
\lim _{\epsilon \rightarrow 0}\left|l_{2 \vec{n}}^{\epsilon, 1}\right|_{2,2 n}^{2}=0
$$

and, if $d H \geq 3 / 2$

$$
\lim _{\epsilon \rightarrow 0} \epsilon^{d-\frac{3}{2 H}}\left|l_{2 \vec{n}}^{\epsilon, 1}\right|_{2,2 n}^{2}=2 T f(H, d, n) .
$$

Suppose now $d H=1$

$$
\begin{gathered}
\left|l_{T, 2 \vec{n}}^{\epsilon, 1}\right|_{2,2 n}^{2}=2 \epsilon^{\frac{1}{2 H}} \int_{0}^{T} d t f(t, H, d, n, \epsilon) \\
f(t, H, d, n, \epsilon) \leq \theta_{t}(\epsilon) \int_{0 \leq x \leq y \leq \theta_{t}(\epsilon)} d x d y \frac{x^{4 H n}}{\left(\left(x^{2 H}+1\right)\left(y^{2 H}+1\right)\right)^{n+d / 2}}
\end{gathered}
$$

and then

$$
\frac{f(t, H, d, n, \epsilon)}{|\log \epsilon|} \leq \theta_{t}(\epsilon)\left\{\frac{a(H, d, n)}{|\log \epsilon|}+b(H, d, n)\right\}
$$

where

$$
a(H, d, n)=\int_{\substack{0 \leq x \leq y \\ x \leq 1}} d x d y \frac{x^{4 H n}}{\left(\left(x^{2 H}+1\right)\left(y^{2 H}+1\right)\right)^{n+d / 2}}
$$

and

$$
b(H, d, n)=\sup _{0 \leq t \leq T} \sup _{\epsilon>0} \frac{1}{|\log \epsilon|} \int_{1 \leq x \leq y \leq \theta_{t}(\epsilon)} d x d y \frac{x^{4 H n}}{\left(\left(x^{2 H}+1\right)\left(y^{2 H}+1\right)\right)^{n+d / 2}} .
$$

So we obtain

$$
\frac{1}{|\log \epsilon|}\left|l_{T, 2 \vec{n}}^{\epsilon, 1}\right|_{2,2 n}^{2} \leq T^{2}\left\{\frac{a(H, d, n)}{|\log \epsilon|}+b(H, d, n)\right\} .
$$

Let us now treat $\left|l_{2 \vec{n}}^{\epsilon, 2}\right|_{2,2 n}^{2}$,

$$
\left|l_{2 \vec{n}}^{\epsilon, 2}\right|_{2,2 n}^{2}=2 \epsilon^{\frac{3}{2 H}-d} \int_{0}^{T} d t g(t, H, d, n, \epsilon)
$$


where

$$
g(t, H, d, n, \epsilon)=\int_{\substack{0 \leq x+y-z \leq \theta_{t}(\epsilon) \\ 0 \leq z \leq x \leq y \leq \theta_{t}(\epsilon)}} d x d y d z \frac{\left(g_{H}(x, y, z)\right)^{2 n}}{\left(\left(x^{2 H}+1\right)\left(y^{2 H}+1\right)\right)^{n+d / 2}}
$$

and

$$
g_{H}(x, y, z)=(x-z)^{2 H}+(y-z)^{2 H}-(x+y-z)^{2 H}-z^{2 H}
$$

we have $\left|g_{H}(x, y, z)\right| \leq 2 x^{2 H}$ and so

$$
\begin{gathered}
z \longmapsto \quad \int_{0 \leq z \leq x \leq y} d x d y \frac{\left(g_{H}(x, y, z)\right)^{2 n}}{\left(\left(x^{2 H}+1\right)\left(y^{2 H}+1\right)\right)^{n+d / 2}}= \\
z^{4 H n+2} \int_{1 \leq x \leq y} d x d y \frac{\left(g_{H}(x, y, 1)\right)^{2 n}}{\left(\left(z^{2 H} x^{2 H}+1\right)\left(z^{2 H} y^{2 H}+1\right)\right)^{n+d / 2}}
\end{gathered}
$$

is well defined on $\mathbb{R}_{+}$and for $d H>3 / 2$, one can choose $\left.\alpha \in\right] 1,2 d H-2[$ such that when $z \rightarrow+\infty$

$$
z^{\alpha} \int_{0 \leq z \leq x \leq y} d x d y \frac{\left(g_{H}(x, y, z)\right)^{2 n}}{\left(\left(x^{2 H}+1\right)\left(y^{2 H}+1\right)\right)^{n+d / 2}} \longrightarrow 0
$$

and then

$$
\int_{\mathbb{R}_{+}} d z \int_{0 \leq z \leq x \leq y} d x d y \frac{\left(g_{H}(x, y, z)\right)^{2 n}}{\left(\left(x^{2 H}+1\right)\left(y^{2 H}+1\right)\right)^{n+d / 2}}=g(H, d, n)<\infty
$$

so that

$$
\lim _{\epsilon \rightarrow 0} g(t, H, d, n, \epsilon)=g(H, d, n) .
$$

Suppose $d H=1$, the same computation as in the case of $\left|l_{2 \vec{n}}^{\epsilon, 1}\right|_{2,2 n}^{2}$ leads to

$$
\frac{1}{|\log \epsilon|}\left|l_{T, 2 \vec{n}}^{\epsilon, 2}\right|_{2,2 n}^{2} \leq 2^{2 n} T^{2}\left\{\frac{a(H, d, n)}{|\log \epsilon|}+b(H, d, n)\right\}
$$

and finally

$$
\frac{1}{|\log \epsilon|}\left|l_{T, 2 \vec{n}}^{\epsilon}\right|_{2,2 n}^{2} \leq 22^{2 n} T^{2}\left\{\frac{a(H, d, n)}{|\log \epsilon|}+b(H, d, n)\right\}
$$


Suppose now $1<d H<3 / 2$, we have

$$
\begin{aligned}
g(t, H, d, n, \epsilon) & \leq \int_{0}^{\theta_{t}(\epsilon)} d z z^{4 H n+2} \int_{1 \leq x \leq y \leq \frac{\theta_{t}(\epsilon)}{z}} d x d y \frac{g_{H}(x, y, 1)}{\left(\left((z x)^{2 H}+1\right)\left((z y)^{2 H}+1\right)\right)^{n+d / 2}} \\
& \leq \int_{0}^{\theta_{t}(\epsilon)} d z z^{2-2 H d} \int_{1 \leq x \leq y} d x d y \frac{2^{2 n} x^{4 H n}}{\left(x^{2 H} y^{2 H}\right)^{n+d / 2}} \\
& =2^{2 n} c(H, d, n) .
\end{aligned}
$$

So that

$$
g(t, H, d, n, \epsilon) \leq 2^{2 n} c(H, d, n)
$$

then

$$
\left|l_{2 \vec{n}}^{\epsilon, 2}\right|_{2,2 n}^{2} \leq \frac{2^{2 n} c(H, d, n)}{(3-2 H d)(2-H d)} T^{4-2 H d}
$$

we know that $\lim _{\epsilon \rightarrow 0}\left|l_{T, 2 \vec{n}}^{\epsilon, 1}\right|_{2,2 n}^{2}=0$, then $\lim _{\epsilon \rightarrow 0}\left|l_{2 \vec{n}}^{\epsilon}\right|_{2,2 n}$ exists.

Finally let us suppose that $d H=3 / 2$

$$
\begin{gathered}
\left|l_{2 \vec{n}}^{\epsilon, 2}\right|_{2,2 n}^{2}=2 \int_{0}^{T} d t g(t, H, d, n, \epsilon) \\
g(t, H, d, n, \epsilon) \leq \int_{0}^{1} d z \int_{z \leq x \leq y \leq \theta_{t}(\epsilon)} d x d y \frac{\left(g_{H}(x, y, z)\right)^{2 n}}{\left(\left(x^{2 H}+1\right)\left(y^{2 H}+1\right)\right)^{n+d / 2}} \\
+\int_{1}^{\theta_{t}(\epsilon)} d z \int_{z \leq x \leq y \leq \theta_{t}(\epsilon)} d x d y \frac{\left(g_{H}(x, y, z)\right)^{2 n}}{\left(\left(x^{2 H}+1\right)\left(y^{2 H}+1\right)\right)^{n+d / 2}} .
\end{gathered}
$$

The first term is bounded by

$$
2^{2 n} \int_{0 \leq x \leq y} d x d y \frac{x^{4 H n}}{\left(\left(x^{2 H}+1\right)\left(y^{2 H}+1\right)\right)^{n+d / 2}}=2^{2 n} e(H, d, n),
$$

the second term is bounded by

$$
\int_{1}^{\theta_{t}(\epsilon)} \frac{d z}{z} \int_{1 \leq x \leq y} \frac{2^{2 n} x^{4 H n}}{\left(x^{2 H} y^{2 H}\right)^{n+d / 2}}=2^{2 n} c(H, d, n) \log \theta_{t}(\epsilon)
$$

so

$$
\frac{1}{|\log \epsilon|}\left|l_{2 \vec{n}}^{\epsilon, 2}\right|_{2,2 n}^{2} \leq \frac{2}{|\log \epsilon|} 2^{2 n} e(H, d, n) T+\frac{2^{2 n}}{H} c(H, d, n) T+\frac{22^{2 n}}{|\log \epsilon|} \int_{0}^{T} \log t d t .
$$


Therefore

$$
\frac{1}{|\log \epsilon|}\left|l_{2 \vec{n}}^{\epsilon}\right|_{2,2 n}^{2}
$$

has a finite non trivial limit and

$$
\lim _{\epsilon \rightarrow 0} \frac{1}{|\log \epsilon|}\left|l_{2 \vec{n}}^{\epsilon}\right|_{2,2 n}^{2} \leq \frac{2^{2 n}}{H} c(H, d, n) T .
$$

\section{References}

[1] Als E. and Nualart N.: Stochastic integration with respect to the fractional Brownian motion. Stochastics and Stochastics Reports 75, 129-152, 2003.

[2] Bolthausen E.: On the construction of the three dimensional polymer measure. Prob. Theory Rel. Fields 97 (1993) 81-101.

[3] Drumond C., de Faria D. and Streit L.: The renormalization of self intersection local times I: The chaos expansion. IDAQP 3 (2000) 223-236.

[4] Drumond C., de Faria D. and Streit L.: The square of self intersection local time of Brownian motion. CMS Conf. Proc. 28 (2000) 115-122.

[5] Dynkin E.B.: Polynomials of the occupation field and related random fields. J. Func. Anal. 58 (1984) 20-52.

[6] Dynkin E.B.: Regularized self-intersection local times of planar Brownian motion. Ann. Prob. 16 (1988) 58-74.

[7] Edwards S.: The statistical mechanics of polymers with excluded volume. Proc. Phys. Sci. 85 (1965) 613-624.

[8] Eddahbi M., Lacayo R., Solé J.L., Vives J. and Tudor C.A.: Regularity and asymtotic behaviour of the local time for the d-dimensional fractional Brownian motion with $N$-parameters. Preprint.

[9] de Faria M., Hida T., Streit L. and Watanabe H.: Intersection local times as generalized white noise functionals. Acta Appl. Math. 46 (1997) 351-362.

[10] Hida T., Kuo H-H., Potthoff J. and Streit L.: White Noise. An Infinite-Dimentional Calculus. Mathematics and its Applications 253. Kluwer-Academic, Dordrecht (1993). 
[11] Imkeller P., Perez-Abreu V. and Vives J.: Chaos expansions of double intersection local time of brownian motion in $\mathbb{R}^{d}$ and renormalization. Stoch. Proc. App. 56 (1995) 1-34.

[12] Imkeller P. and Yan J-A.: Multiple intersection local time of planar Brownian motion as a particular Hida distribution. J. Func. Anal. 140 (1996) 256-273.

[13] Kuo H-H.: Donker's delta function as a generalized Brownian functional and its application. Lecture Notes in Control and Information Sciences, 49 (1983) 167-178.

[14] Le Gall J.F.: Sur le temps local d'intersection du mouvement Brownian plan et la méthode de renormalization de Varadhan. L.N.M 1123 314-331. Springer, Berlin (1985).

[15] Lévy P.: Le mouvement brownien plan. Amer. J. Math. 62 (1940) 440$48 \%$.

[16] Nualart D.: Stochastic integration with respect to the fractional Brownian motion and applications. Preprint.

[17] Nualart D. and Vives J.: Chaos expansion and local times. Publ. Math. 36(2) (1992) 827-836.

[18] Rezgui A. and Streit L.: The renormalization of self intersection local times of Brownian motion. Preprint.

[19] Rosen J.: A local time approach to the self-intersections of Brownian paths in space. Comm. Math. Phys. 88 (1983) 327-338.

[20] Rosen J.: Tanaka's formula and renormalization for intersections of planar Brownian motion. Ann. Prob. 14 (1986) 1245-1251.

[21] Rosen J.: The intersection local time of fractional Brownian motion in the plane. J. Multi. Analy. 23 (1987) 37-46.

[22] Symanzik K.: Euclidean Quantum Field Theory, in R. Jost ed., Local Quantum Theory. Academic, New York. (1969).

[23] Varadhan S.R.S.: Appendix to "Euclidean Quantum Field theory" by Szymanzik K., in: R. Jost ed., local Quantum Theory. Academic, New York (1969). 
[24] Watanabe H.: The local time of self-intersections of Brownian motions as a generalized Brownian functionals. Lett. Math. Phy. 23 (1991) 1-9.

[25] Westwater M. J.: On Edwards' model for long polymer chains. Commun. Math. Phys. 72 (1980) 131-174.

[26] Wolpert R.: Weiner path intersections and local time. J. Func. Anal. 30 (1978) 329-340.

[27] Wolpert R.: Local time and a particle picture for Euclidean field theory. J. Func. Anal. 30 (1978) 341-35\%.

[28] Yor M.: Renormalization et convergence en loi pour les temps locaux d'intersections du mouvement brownien dans $\mathbb{R}^{3}$. L.N.M 1123 350-365. Springer, Berlin (1985).

Received: October 27, 2006 